\title{
Diseño de una Guía Didáctica basada en la Integración de Mundos Virtuales al Entorno Educativo de la Universidad de Cundinamarca
}

\author{
Geovanny A. Martínez \\ Universidad de Cundinamarca, Facultad de Ciencias Administrativas, Económicas y Contables, \\ Programa de Administración de Empresas, Fusagasugá - Colombia. (e-mail: g-andres5@hotmail.com)
}

Recibido Jun. 20, 2016; Aceptado Ago. 31, 2016; Versión final Oct. 19, 2016, Publicado Feb. 2017

\begin{abstract}
Resumen
En este artículo se propone el diseño de una guía didáctica basada en la integración de mundos virtuales al entorno educativo de la Universidad de Cundinamarca Sede Fusagasugá con el propósito de fomentar la colaboración entre docentes y alumnos. Para ello se emplearon cuatro etapas metodológicas: i) definir el perfil de usuario; ii) realizar un acercamiento a los mundos virtuales mediante una presentación; iii) aplicar un cuestionario exploratorio; y iv) identificar los parámetros de diseño. Como resultado se produjo la guía didáctica caracterizada y fundamentada en la colaboración y la creatividad. La aplicación y valoración de la guía por parte de los usuarios permitió detectar que el nivel de facilidad de uso y utilidad es satisfactorio. Se concluye que la guía promueve el aprendizaje colaborativo y se convierte en modelo para la integración de otras herramientas colaborativas al entorno de la educación superior.
\end{abstract}

Palabras clave: guía didáctica; mundos virtuales; entorno educativo; colaboración entre docentes y alumnos; aprendizaje colaborativo

\section{Design of a Pedagogical Guide based on the Integration of Virtual Worlds into the Educational Environment of the University of Cundinamarca}

\begin{abstract}
This article describes the design of a pedagogical guide based on the integration of virtual worlds into the educational environment of the University of Cundinamarca Fusagasugá branch, in order to promote collaboration between teachers and students. Four methodological steps were employed to achieve this: i) defining the user's profile; ii) making an approach for the teachers to virtual worlds through a presentation; iii) applying an exploratory survey; and iv) identifying the design parameters. This resulted in a pedagogical guide characterized and based on collaboration and creativity. Implementation and evaluation of this guide by the users allowed detecting that the level of easiness and usefulness is satisfactory. It is concluded that the guide promotes collaborative learning and becomes a model for the integration of other collaborative tools into higher education environments.
\end{abstract}

Keywords: pedagogical guide; virtual worlds; educational environment; collaboration between teachers and students; collaborative learning 


\section{INTRODUCCIÓN}

Desde hace algún tiempo se visualiza un gran potencial en el uso de entornos virtuales para la enseñanza y el aprendizaje, Wilson (Citado en Calzadilla, 2002) explica que los entornos de aprendizaje son lugares donde los alumnos trabajan juntos, ayudándose unos a otros, utilizando una gama de instrumentos y recursos informativos que permiten la búsqueda de los objetivos de aprendizaje y la solución de problemas; estos entornos sustentan su soporte pedagógico en el paradigma constructivista (Lizarralde y Huapaya, 2012) en el que los alumnos tienen un papel activo en su aprendizaje, pues no sólo absorben información, sino que también relacionan los conocimientos asimilados previamente para construir unos nuevos (Huang et al., 2010), además se pueden utilizar para facilitar las tareas de aprendizaje que conducen a la mejora de la transferencia de conocimientos y habilidades a situaciones reales a través de la contextualización del aprendizaje.

El uso de entornos colaborativos destinados a la enseñanza no es un concepto nuevo, puesto que, en los programas educativos de nivel superior abundan los cursos montados en soportes que constituyen entornos virtuales de aprendizaje (Chibás-Ortíz et al., 2014), sin embargo, la ubicuidad de las comunidades de colaboración en línea y el uso de los mundos virtuales a gran escala, junto con el creciente poder de la tecnología informática, han aumentado el potencial del aprendizaje colaborativo de una manera que antes no era posible (Whitton y Hollins, 2008). Para Dewey (Citado en Salinas y Benito, 2008) el núcleo de la enseñanza consiste en diseñar ambientes donde los alumnos pueden interactuar y estudiar la forma de aprender; reconociendo que los mundos virtuales hacen parte de esos ambientes, adquieren gran relevancia pedagógica porque en este tipo de entornos los estudiantes están propensos a explorar, participar, descubrir nuevos conocimientos y desarrollar habilidades con una mayor motivación y autonomía (Dreher et al., 2009). Desde un enfoque constructivista del aprendizaje y dentro de estas comunidades, los estudiantes tienen la posibilidad de descubrir contenido y crear conexiones significativas a través de la creatividad y la imaginación (Coffman y Klinger, 2007).

En el año 2007 el Informe Horizon clasificó los mundos virtuales como un área emergente que podía afectar a la educación superior. En el año 2008, la primera edición de la versión de este informe para Australia y Nueva Zelanda señaló que los mundos virtuales tendrían un tiempo probable de adopción de un año o menos y durante ese mismo año en el Reino Unido se publicó un informe explicando diversas formas en que los mundos virtuales inmersivos 3D estaban siendo utilizados para el aprendizaje, entre ellas se incluían tutorías, exploraciones y juegos de rol. Asimismo y producto de otro estudio que inició en el año 2009 en Australia y Nueva Zelanda acerca de la situación de los mundos virtuales en la educación superior se demostró que hubo un aumento sostenido en su uso y que Second Life era el más utilizado seguido de ActiveWorlds 3D, también se presagió que la adopción de los mundos virtuales por académicos mayores de cuarenta y seis años iría en aumento (Dalgarno et al., 2011).

En la actualidad, varios campus universitarios están llevando a cabo las clases y la formación en entornos virtuales 3D (Moldoveanu et al., 2014). Más de cien universidades en los Estados Unidos y en otros países poseen o alquilan tierra virtual en Second Life, por ejemplo, el campus virtual de la Universidad de Princeton acoge actuaciones musicales, el campus de la Universidad de Carolina del Norte alberga una clínica virtual, la Universidad de Kentucky posee una biblioteca (Baker et al., 2009), la Escuela de Derecho de Harvard ha creado tribunales simulados (Campbell, 2009), La Universidad Tecnológica de Nanyang en Singapur cuenta con un campus virtual en donde se les ayuda a los estudiantes extranjeros a adaptarse al campus real (Hew y Cheung, 2010). En Latinoamérica las instituciones educativas han integrado los mundos virtuales a sus procesos académicos, ejemplo de ello es la Universidad de San Carlos en Guatemala (USAC) que a partir de estudios de casos refleja el significado pedagógico que se manifiesta en estos espacios virtuales 3D (Quinche y González, 2011), del mismo modo la Universidad Nacional Autónoma de México (UNAM) y la Universidad San Martín de Porres de Perú (USMP) cuentan con un campus en Second Life para explorar el potencial de este mundo virtual.

En el caso de Colombia la incorporación de esta tecnología a las instituciones de educación superior apenas se está desarrollando y los casos más sobresalientes se limitan a Second Life, se destacan la Universidad del Rosario que posee una isla denominada Calatrava en donde se realizan simulaciones; la Pontificia Universidad Javeriana que ofrece talleres dedicados a la creación de espacios dentro del mundo virtual; la Corporación Universitaria Minuto de Dios (UNIMINUTO) en la que se propuso el diseño y puesta en marcha de un prototipo de campus virtual y que después de su ejecución indicó entre otros aspectos que el nivel de generación de conocimiento es más alto que con las clases tradicionales (Quinche y González, 2011).

A partir de la acogida que han tenido los mundos virtuales en la educación superior se puede afirmar que estas plataformas ofrecen una oportunidad para rediseñar los enfoques pedagógicos en lugar de 
simplemente reproducir los métodos de enseñanza tradicionales (Dreher et al., 2009), pero es necesario resaltar que en estos escenarios se han de generar propuestas curriculares y didácticas flexibles que se adapten a las características y capacidades del alumno, profundizando y estimulando la indagación y la autonomía (Salinas y Lizana, 2014). Jacobson (Citado en Dalgarno y Lee, 2010) acude a varios estudios llevados a cabo en la década de los 90 , cuyos resultados sugieren que la inmersión en entornos virtuales de aprendizaje 3D, si se usan apropiadamente proporciona valor añadido. De otro lado, Fetscherin y Lattemann (2008) sustentan que debido a la naturaleza de estas tecnologías emergentes se necesita más investigación para entender mejor el comportamiento del usuario y sugieren que los mundos virtuales distintos de Second Life deben tenerse en cuenta para entender completamente ese comportamiento y la intención de utilizar este tipo de aplicaciones; por lo tanto la investigación futura debe incluir estudios para establecer la validez de los supuestos sobre los entornos virtuales de aprendizaje 3D (Dalgarno y Lee, 2010).

Entendiendo que los educadores necesitan explorar continuamente nuevas estrategias para involucrar a los estudiantes y para crear entornos que mezclen tecnología y métodos de colaboración (Coffman y Klinger, 2007), y que los mundos virtuales se conciben como una estrategia para vencer el aislamiento que experimentan los alumnos en el aula de clase y que promueven la realización de actividades en grupo para generar conocimiento, este artículo plantea el diseño de una guía didáctica basada en la integración de los mundos virtuales al entorno educativo de la Universidad de Cundinamarca Sede Fusagasugá con el fin de fomentar la colaboración entre docentes y alumnos del Programa de Administración de Empresas. Se utiliza un enfoque descriptivo ya que tiene en cuenta las experiencias, expectativas y opiniones de los docentes hacia los mundos virtuales mediante métodos como el cuestionario y el test. Para poder diseñar adecuadamente la guía didáctica se propone en primera instancia percibir la apreciación de los docentes en lo referente al conocimiento y expectativas del uso de los mundos virtuales como herramientas colaborativas, luego se procede a estructurar el contenido de la guía de manera tal que sea comprensible y replicable en otros programas de educación superior; una vez diseñada la guía didáctica se evalúa la utilidad y facilidad de uso de la misma.

\section{MATERIALES Y MÉTODOS}

El propósito del trabajo es el diseño de una guía didáctica basada en la integración de los mundos virtuales al entorno educativo de la Universidad de Cundinamarca Sede Fusagasugá, para ello se establecieron las siguientes etapas metodológicas: 1) Definición del perfil de usuario; 2) Acercamiento a mundos virtuales; 3) Cuestionario exploratorio; 4) Identificación de parámetros de la guía didáctica.

\section{Definición del Perfil de Usuario}

Inicialmente se tuvo en cuenta a la totalidad de docentes pertenecientes al Programa de Administración de Empresas y que se clasifican en tres modalidades tal como se refleja en la Tabla 1.

Tabla 1: Número de Docentes Según Modalidad

\begin{tabular}{|l|c|}
\hline Modalidad & Cantidad de Docentes \\
\hline Tiempo Completo Ocasional & 10 \\
\hline Medio Tiempo Ocasional & 4 \\
\hline Hora Cátedra & 16 \\
\hline Total & 30 \\
\hline
\end{tabular}

Al definir el perfil de usuario se contemplaron tres criterios: Carga académica, Disponibilidad de tiempo e Interés por la temática. Por tanto, se eligió a los Docentes Tiempo Completo Ocasional como usuarios por tener una carga académica más alta en comparación con los otros docentes; por la plena disposición de tiempo y compromiso para llevar a cabo las etapas propuestas y por demostrar un interés generalizado en la integración de los mundos virtuales al proceso educativo.

\section{Acercamiento a Mundos Virtuales}

Para dar a conocer a los usuarios una visión general sobre los mundos virtuales, se realizó una presentación denominada: "Mundos Virtuales en la Educación". En primer lugar se explicó la importancia de utilizar estos entornos en el proceso educativo, luego se dio a conocer las características y se describieron los mundos más populares según distintas áreas. Finalmente se analizaron los beneficios de tres mundos virtuales que han incursionado en el ámbito educativo: ActiveWorlds 3D, Second Life y The Education District. Adicionalmente se realizó la instalación y exploración de los tres mundos virtuales y los docentes se familiarizaron con estos entornos. 

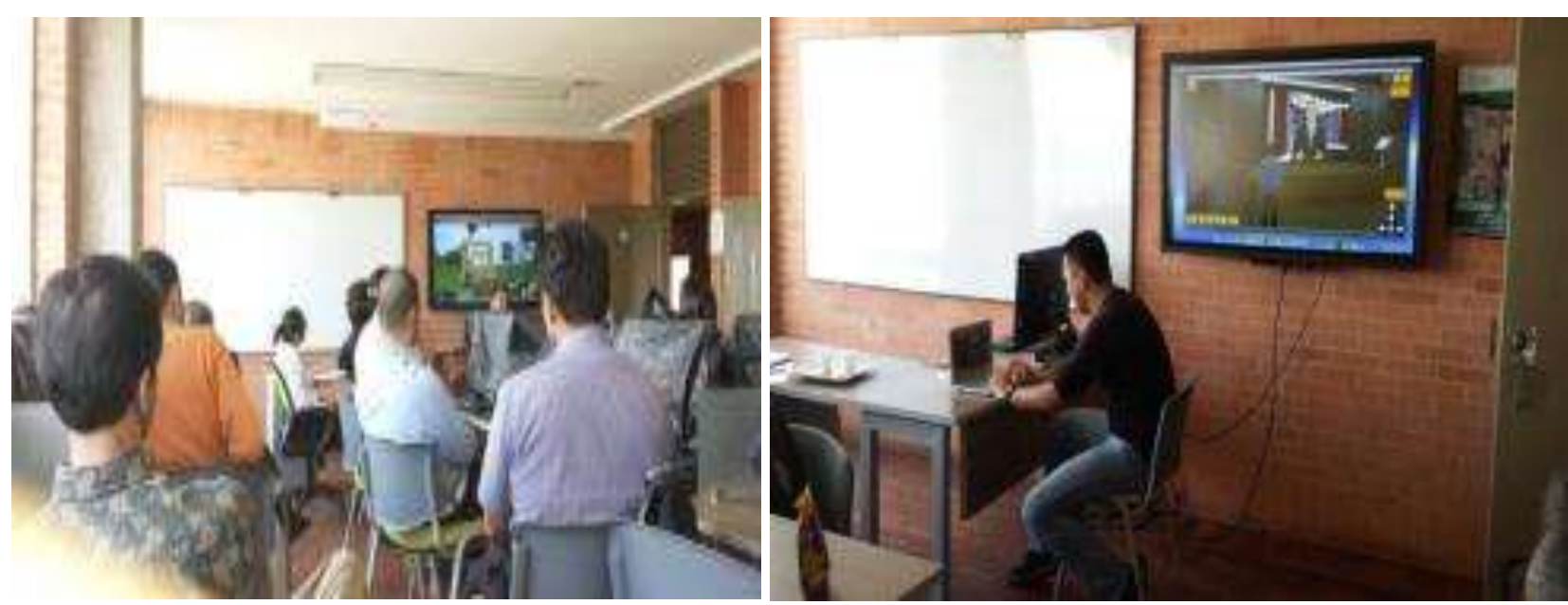

Fig. 1: Presentación Acercamiento a Mundos Virtuales
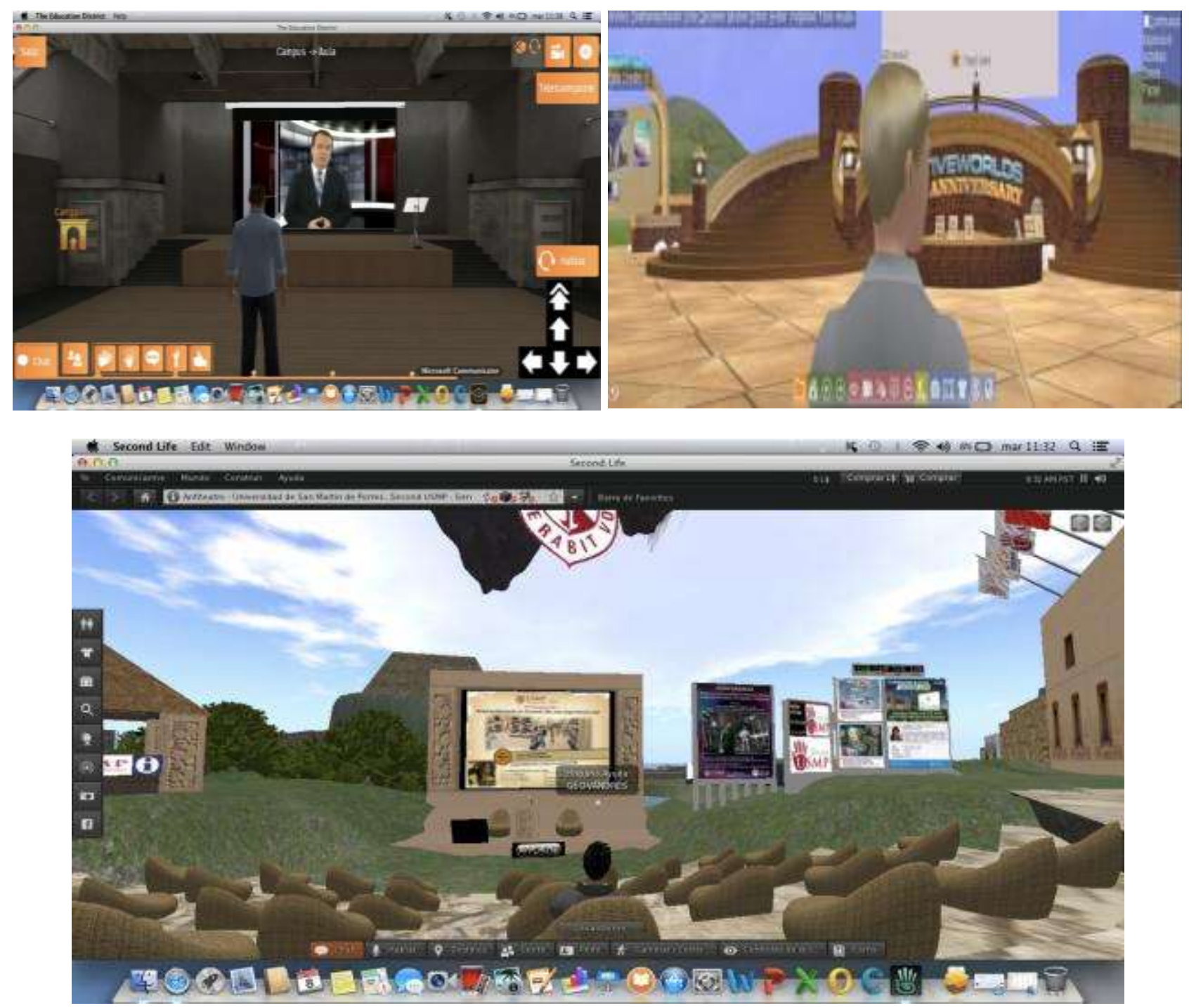

Fig. 2: Exploración en TED, ActiveWorlds y Second Life

\section{Cuestionario Exploratorio}

Con el fin de identificar la percepción y el conocimiento sobre el uso de los mundos virtuales y además las expectativas acerca de la guía didáctica, se diseñó y aplicó a los Docentes Tiempo Completo Ocasional del Programa de Administración de Empresas de la Universidad de Cundinamarca Sede Fusagasugá un cuestionario exploratorio cuyos resultados se presentan en la Figura 3. 


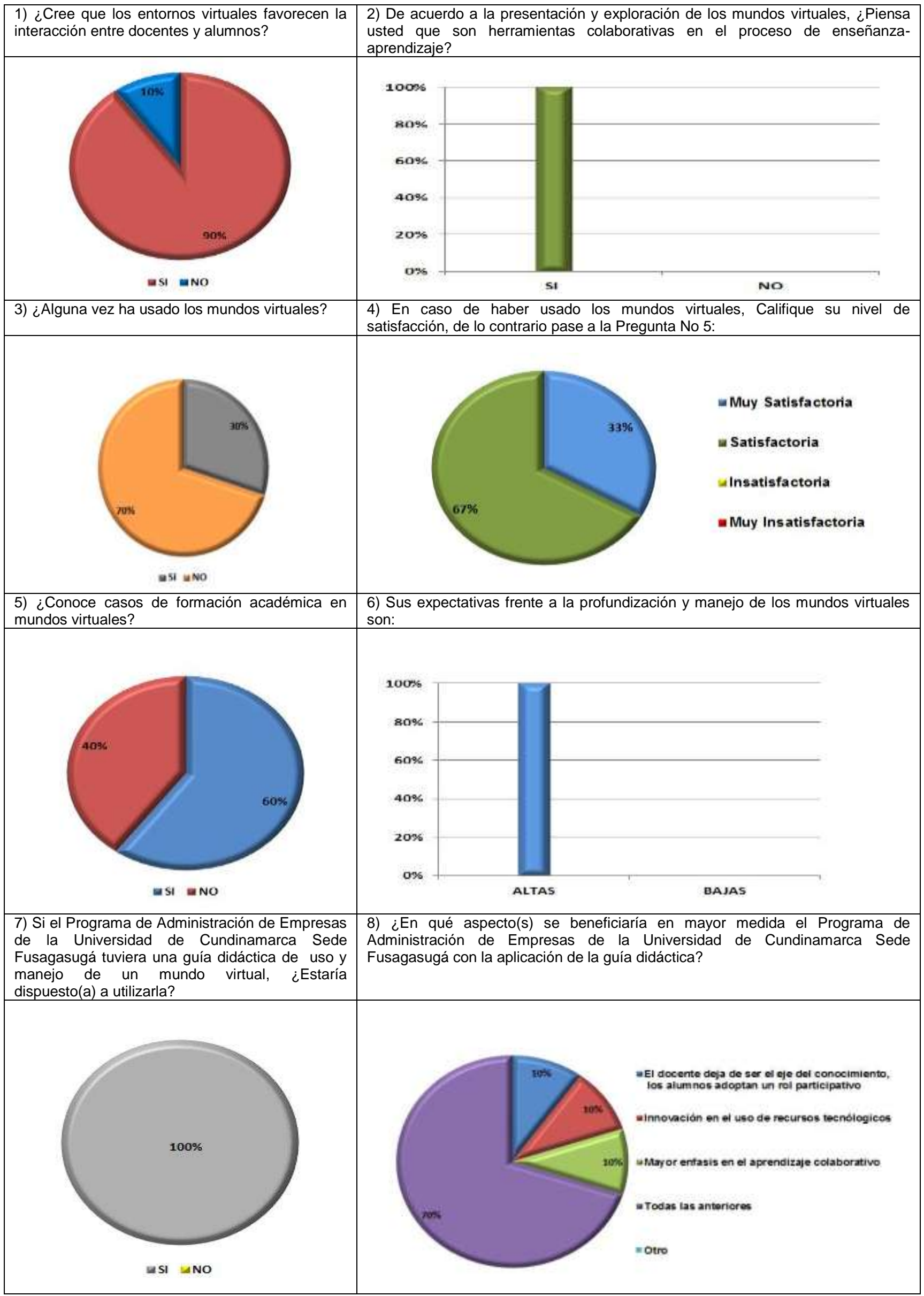

Fig. 3: Resultados Cuestionario Exploratorio 
De los resultados obtenidos en el cuestionario exploratorio se establece que nueve docentes creen que los entornos virtuales favorecen la interacción con los alumnos; los diez docentes consideran a los mundos virtuales como una herramienta colaborativa de acuerdo a la presentación y exploración realizada; tres docentes alguna vez han usado los mundos virtuales pero no necesariamente con fines educativos sino por curiosidad o diversión y de estos, dos manifiestan haber tenido una experiencia satisfactoria mientras uno dice haber tenido una experiencia muy satisfactoria; a su vez seis docentes expresan conocer casos de formación académica dentro de los mundos virtuales en otras instituciones y los diez docentes coinciden en que las expectativas frente a la profundización y manejo de los mundos virtuales son altas. De otra parte, al plantear la posibilidad de que el Programa de Administración de Empresas de la Universidad de Cundinamarca sede Fusagasugá tuviera una guía didáctica de uso y manejo de un mundo virtual, la totalidad de los docentes estarían dispuestos a utilizarla. Finalmente siete docentes sostienen que los aspectos que beneficiarían en mayor medida al programa con la aplicación de la guía didáctica son en su conjunto: El docente deja de ser el eje del conocimiento; Innovación en el uso de recursos tecnológicos; Mayor énfasis en el aprendizaje.

De igual manera se les solicito a los docentes que valoraran de 1 a 5 (siendo 1 el nivel mínimo y 5 el nivel máximo) a los mundos virtuales ActiveWorlds 3D, Second Life y The Education District de acuerdo a la instalación y exploración realizadas, para así determinar a cuál de ellos se le iba a diseñar la guía didáctica. La puntuación correspondiente a los tres mundos virtuales por el grupo de usuarios se ve reflejada en la Tabla 2.

Tabla 2: Puntuación Mundos Virtuales

\begin{tabular}{|c|c|c|c|}
\hline \multirow{2}{*}{ Usuarios } & \multicolumn{3}{|c|}{ Mundos Virtuales y Puntuación } \\
\cline { 2 - 4 } & Active Worlds & Second Life & TED \\
\hline 1 & 4 & 4 & 5 \\
\hline 2 & 3 & 3 & 5 \\
\hline 3 & 3 & 4 & 4 \\
\hline 4 & 5 & 5 & 5 \\
\hline 5 & 3 & 3 & 5 \\
\hline 6 & 4 & 4 & 5 \\
\hline 7 & 5 & 5 & 5 \\
\hline 8 & 5 & 5 & 5 \\
\hline 9 & 5 & 4 & 2 \\
\hline 10 & 4 & 4 & 5 \\
\hline Total Puntos & 41 & 41 & 46 \\
\hline
\end{tabular}

El mundo virtual que obtuvo la mayor puntuación fue The Education District con un total de 46 puntos, mientras que ActiveWorlds 3D y Second Life obtuvieron un total de 41 puntos cada uno. Lo que significa que los usuarios prefirieron al mundo virtual The Education District para el diseño de la guía didáctica.

\section{Identificación de Parámetros de la Guía Didáctica}

Una vez definido que The Education District (TED) es el mundo virtual escogido por los usuarios, se empleó la técnica del Brainwriting para establecer los parámetros a tener en cuenta en la guía didáctica. Dicha técnica es una variante del Brainstorming que se realiza por escrito y se usa para obtener una serie de ideas en torno a una situación planteada. El Brainwriting es útil porque al leer las anotaciones anteriores cada persona reacciona creativamente al momento de generar nuevas ideas. Otra de las ventajas de esta técnica es la libertad que se le da a la imaginación pues el hecho de escribir en un papel lo que se piensa en vez de hablar en público fortalece el potencial creativo sobre todo en aquellas personas que no integran grupos de trabajo con frecuencia para este tipo de ejercicios.

En esta ocasión se utilizó la variable con una sola hoja, es decir un miembro del grupo plasmó una idea de cómo desearía que fuera la guía didáctica, esta hoja se pasó al siguiente miembro quien leyó la idea anterior y plasmó su propia idea. Así, se rotó la hoja usuario por usuario hasta llegar al último miembro y se recolectaron las ideas del grupo para afinar los insumos de diseño de la guía didáctica. (Fig. 4). 


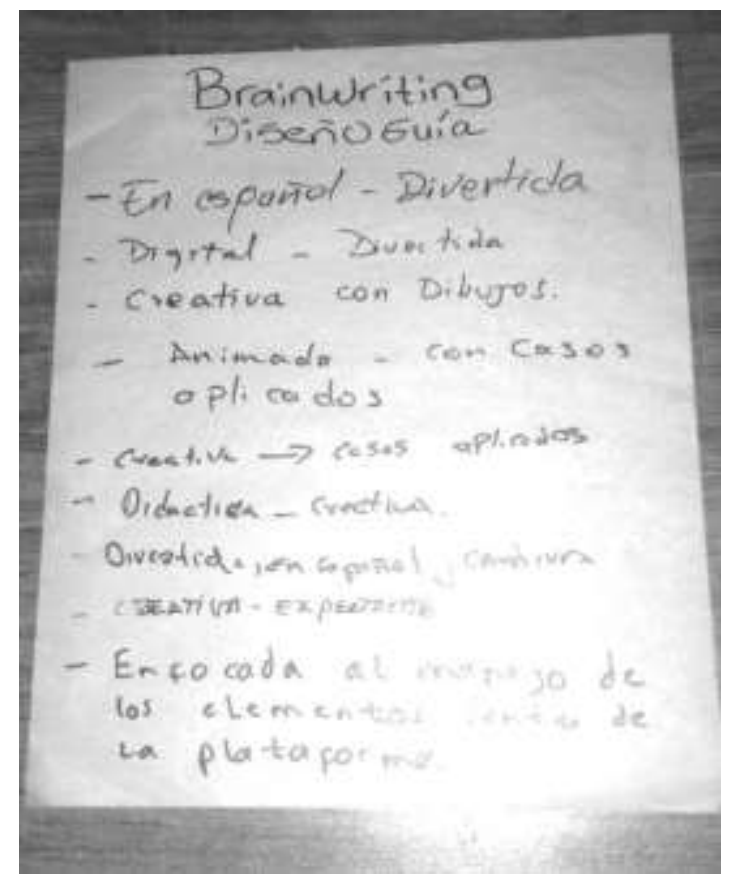

Fig. 4: Brainwriting Diseño Guía Didáctica

\section{RESULTADOS}

Dando cumplimiento al objetivo del presente artículo, se desarrolló una guía didáctica basada en la integración del mundo virtual The Education District al entorno educativo de la Universidad de Cundinamarca Sede Fusagasugá. La guía cuenta con las siguientes características: Elaborada en formato pdf; Fundamentada en la creatividad, colaboración y personalización; Incluye los requerimientos técnicos dependiendo del sistema operativo (Fig. 5).

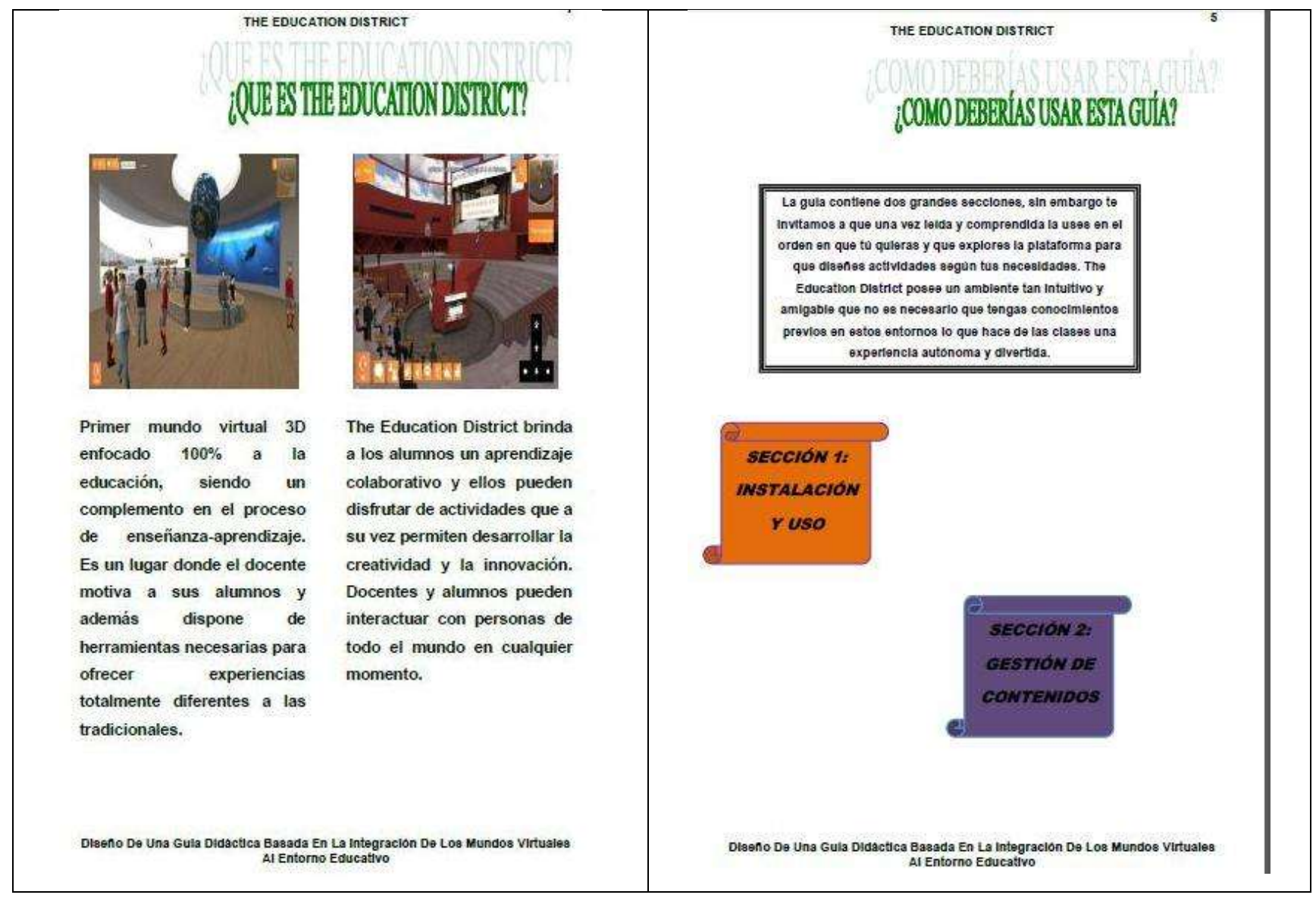

Fig. 5: Apartados de la Guía Didáctica 
La guía consta de dos secciones: 1) Instalación y uso del visor: Inicialmente se presenta la forma de descargar el programa para las distintas plataformas (iphone, ipad, Mac, Windows), mediante la página oficial The Education District (http://www.theeducationdistrict.com/es/). De otro lado, se orienta al lector para que desde la dirección de acceso (http://public.theeducationdistrict.com/) pueda crear una cuenta introduciendo un usuario y una contraseña. Por último se describe como configurar la interfaz, elegir el avatar y explorar cada uno de los escenarios que constituyen el mundo virtual. 2) Gestión de contenidos: The Education District permite administrar contenidos 2D y 3D, programar clases y crear eventos en cada uno de los escenarios por medio de diversos roles (Managers, Publishers, Admins, Shop Managers) en un espacio denominado Zona de Gestión (http://cms.theeducationdistrict.com/). Esta sección enseña al usuario a manejar las distintas funcionalidades para cada uno de los roles mencionados. De conformidad con lo descrito y para mayor conocimiento, la guía didáctica se encuentra disponible para su consulta en el repositorio del sistema de gestión bibliotecaria de la Universidad de Cundinamarca (http://dspace.unicundi.edu.co:8080/xmlui/handle/123456789/188).

\section{Aplicación de Guía Didáctica}

Finalizada la guía, esta se entregó en formato digital a cada usuario para ser leída y que posteriormente la pusieran en práctica e interactuaran con el mundo virtual The Education District sin la ayuda de ningún otro usuario ni facilitador. Se propuso entonces unas tareas que debían ser ejecutadas: Descargar e instalar el visor The Education District; Crear una cuenta y loguearse con usuario y contraseña; Escoger un avatar y realizar las configuraciones pertinentes en cuanto a entrada y salida de sonido y calidad de imagen; Visitar cada uno de los escenarios y explorarlos utilizando las acciones propias del avatar; Loguearse en la zona de gestión teniendo en cuenta que previamente se creó un acceso para cada usuario con el rol Admins; Crear un Contenido 2D; Crear Contenidos 3D (NPC o personaje animado, Punto de Información, Presentación) y Programar un Evento.

\section{Medición de Facilidad de Uso y Utilidad}

Para conocer las opiniones y valoraciones acerca de la guía didáctica luego de la realización de las tareas propuestas se recurrió al test como medida de usabilidad donde se evalúa la interacción de los usuarios llevando a cabo tareas reales, por ello se presentaron dos tipos de test para ser aplicados, uno de facilidad de uso percibida como el grado en que la persona cree que con el uso de una herramienta estará libre de esfuerzo y otro de utilidad que se refiere al grado en que la persona cree que el uso de esa herramienta puede mejorar su rendimiento en el trabajo (Fetscherin y Lattemann, 2008).

En el primer test se les solicito a los usuarios que de acuerdo a las indicaciones suministradas por la guía didáctica calificaran de 1 a 5 el nivel de facilidad (siendo 1 el nivel mínimo y 5 el nivel máximo) en la ejecución de las siguientes actividades: Descarga e instalación del visor The Education District; Exploración por el mundo virtual; Elección del Avatar y configuración de la interfaz; Teletransporte por cada uno de los escenarios; Realización de las acciones propias de un avatar (correr, sentarse, chatear, hablar etc.); Acceso a los recursos dispuestos dentro de los escenarios (juegos, zonas de diversión etc.); Interacción con NPCs, Puntos de Información y otros elementos; Manejo de la zona de gestión; Planeación de clases y eventos; Creación de Contenidos 2D y 3D.

En el segundo test se les solicito a los usuarios que calificaran de 1 a 5 el nivel de acuerdo (donde 1 representa "Totalmente en Desacuerdo" y 5 representa "Totalmente de Acuerdo") con las siguientes afirmaciones: El uso de la guía cumple con las necesidades y expectativas; Las explicaciones de la guía didáctica son claras y pertinentes; Es sencillo seguir las indicaciones que proporciona la guía; Los elementos que contiene la guía son los necesarios para usar y manejar el mundo virtual; Socializar la guía con otros usuarios es apropiado para el aprendizaje; El lenguaje utilizado en la guía es comprensible; La guía didáctica en su conjunto es agradable y amena; Aprender a manejar el mundo virtual a través de la guía didáctica es un método acertado; Poner en practica la guía con frecuencia fomenta la interacción y colaboración entre docentes y alumnos; La guía es un modelo para la integración de entornos colaborativos al sistema educativo. La puntuación dada por los usuarios tanto para el test de facilidad de uso como para el test de utilidad se encuentra en la Tabla 3 y Tabla 4 respectivamente.

Según la puntuación del test de facilidad de uso y al seguir las pautas proporcionadas por la guía didáctica se observa que para dos usuarios la descarga e instalación del visor fue muy fácil (5), para cuatro usuarios fue relativamente fácil (3), para tres usuarios fue fácil (4) y a un usuario se le complico el proceso (2). Un usuario tuvo dificultad en la exploración por los escenarios (2), para siete usuarios fue fácil la exploración (4) y a dos usuarios les pareció muy fácil (5). La elección del avatar y la configuración de la interfaz fue fácil para tres usuarios (4), para seis usuarios fue muy fácil (5) y un usuario presentó dificultad en los ajustes del sonido (2). El teletransporte fue fácil para tres usuarios (4) y para siete resultó muy fácil (5). Acciones como 
sentarse, aplaudir y hablar fueron muy fáciles para dos usuarios (5), para seis usuarios fueron fáciles (4) y para dos usuarios fueron difíciles (2). Para un usuario el acceso a los recursos dispuestos dentro de los escenarios fue muy fácil (5), para cuatro usuarios fue fácil (4), para otro tanto fue relativamente fácil (3) pero para un usuario fue difícil (2). La interacción con elementos animados fue difícil para un usuario (2), para seis usuarios fue relativamente fácil (3), para dos usuarios fue fácil (4) y para un usuario fue muy fácil (5). El manejo de la zona de gestión fue difícil para dos usuarios (2), para seis usuarios el manejo de esta zona fue relativamente fácil (3), mientras que para un usuario fue fácil (4) y para otro muy fácil (5). La planeación de clases y programación de eventos fue muy fácil para dos usuarios (5), para seis usuarios fue relativamente fácil (3) y para dos usuarios fue fácil (4). La creación de contenidos 2D y 3D fue fácil para tres usuarios (4), relativamente fácil para seis (3) y difícil para un usuario (2).

Tabla 3: Puntuación Test Facilidad de Uso

\begin{tabular}{|l|c|c|c|c|c|c|c|c|c|c|}
\hline \multicolumn{1}{|c|}{ Aspecto } & \multicolumn{5}{c|}{ Puntuación Por Usuario } \\
\cline { 2 - 9 } & 1 & 2 & 3 & 4 & 5 & 6 & 7 & 8 & 9 & 10 \\
\hline Descarga e instalación del visor The Education District & 3 & 4 & 3 & 5 & 3 & 4 & 2 & 3 & 5 & 4 \\
\hline Exploración por el mundo virtual & 2 & 4 & 4 & 4 & 4 & 5 & 4 & 4 & 5 & 4 \\
\hline Elección del Avatar y configuración de la interfaz & 4 & 5 & 2 & 4 & 5 & 5 & 4 & 5 & 5 & 5 \\
\hline Teletransporte por cada uno de los escenarios & 5 & 5 & 5 & 5 & 4 & 5 & 4 & 4 & 5 & 5 \\
\hline Realización de las acciones propias de un avatar & 4 & 4 & 2 & 4 & 5 & 4 & 2 & 4 & 5 & 4 \\
\hline Acceso a los recursos dispuestos dentro de los escenarios & 3 & 4 & 3 & 4 & 3 & 4 & 2 & 3 & 5 & 4 \\
\hline Interacción con NPCs, Puntos de Información y otros elementos & 3 & 4 & 3 & 4 & 3 & 3 & 3 & 2 & 5 & 3 \\
\hline Manejo de la zona de gestión & 3 & 3 & 2 & 4 & 3 & 3 & 2 & 3 & 5 & 3 \\
\hline Planeación de clases y eventos dentro del mundo virtual & 3 & 3 & 3 & 4 & 3 & 3 & 3 & 4 & 5 & 5 \\
\hline Creación de Contenidos 2D y 3D & 3 & 3 & 3 & 4 & 3 & 3 & 2 & 3 & 4 & 4 \\
\hline
\end{tabular}

Tabla 4: Puntuación Test Utilidad

\begin{tabular}{|c|c|c|c|c|c|c|c|c|c|c|}
\hline \multirow{2}{*}{ Afirmaciones } & \multicolumn{10}{|c|}{ Puntuación Por Usuario } \\
\hline & 1 & 2 & 3 & 4 & 5 & 6 & 7 & 8 & 9 & 10 \\
\hline El uso de la guía cumple con las necesidades y expectativas & 4 & 4 & 5 & 5 & 5 & 4 & 4 & 5 & 5 & 5 \\
\hline Las explicaciones de la guía didáctica son claras y pertinentes & 4 & 5 & 4 & 5 & 4 & 4 & 3 & 3 & 5 & 4 \\
\hline Es sencillo seguir las indicaciones que proporciona la guía & 5 & 4 & 3 & 4 & 4 & 3 & 3 & 3 & 5 & 4 \\
\hline $\begin{array}{l}\text { Los elementos que contiene la guía son los necesarios para usar y } \\
\text { manejar el mundo virtual }\end{array}$ & 5 & 5 & 5 & 5 & 5 & 5 & 5 & 5 & 5 & 5 \\
\hline $\begin{array}{l}\text { Socializar la guía con otros usuarios es apropiado para el } \\
\text { aprendizaje }\end{array}$ & 4 & 4 & 4 & 5 & 5 & 3 & 4 & 5 & 5 & 5 \\
\hline El lenguaje utilizado en la guía es comprensible & 5 & 4 & 4 & 5 & 5 & 4 & 3 & 3 & 5 & 4 \\
\hline La guía didáctica en su conjunto es agradable y amena & 5 & 5 & 5 & 5 & 5 & 5 & 5 & 5 & 5 & 5 \\
\hline $\begin{array}{l}\text { Aprender el manejo del mundo virtual a través de la guía didáctica } \\
\text { es un método acertado }\end{array}$ & 5 & 4 & 4 & 4 & 5 & 5 & 4 & 5 & 5 & 5 \\
\hline $\begin{array}{l}\text { Poner en practica la guía con frecuencia fomenta la interacción y } \\
\text { colaboración entre docentes y alumnos }\end{array}$ & 5 & 4 & 5 & 4 & 5 & 4 & 5 & 5 & 5 & 4 \\
\hline $\begin{array}{l}\text { La guía es un modelo para la integración de entornos colaborativos } \\
\text { al sistema educativo }\end{array}$ & 4 & 4 & 5 & 4 & 5 & 4 & 5 & 5 & 5 & 4 \\
\hline
\end{tabular}

En lo que tiene que ver con el test de utilidad, cuatro usuarios expresaron estar de acuerdo con el cumplimiento de necesidades y expectativas por parte de la guía didáctica (4), mientras que seis usuarios estuvieron totalmente de acuerdo (5). Dos usuarios estuvieron parcialmente de acuerdo con la claridad y pertinencia de las explicaciones de la guía (3), cinco usuarios expresaron estar de acuerdo (4) y tres usuarios valoraron con la puntuación máxima este ítem (5). Cuatro usuarios manifestaron estar de acuerdo 
con la sencillez de las indicaciones de la guía (4), dos usuarios dieron la máxima puntuación (5), en cambio para cuatro usuarios las indicaciones fueron apenas sencillas (3). Para la totalidad de usuarios los elementos que contiene la guía son los necesarios para usar y manejar el mundo virtual (5). Los usuarios valoraron que el hecho de socializar la guía con otros es apropiado para el aprendizaje, cinco usuarios estuvieron totalmente de acuerdo (5) y cuatro estuvieron de acuerdo (4), un usuario estuvo parcialmente de acuerdo con esta afirmación (3). Cuatro usuarios estuvieron de acuerdo con que la guía utiliza un lenguaje comprensible (4), otros cuatro estuvieron totalmente de acuerdo (5) y para dos usuarios el lenguaje fue apenas comprensible (3). Los usuarios estuvieron totalmente de acuerdo con que la guía didáctica es agradable y amena (5) y coincidieron con que el método más acertado para aprender a manejar el mundo virtual es a través de la guía, seis usuarios estuvieron totalmente de acuerdo con esta afirmación (5) y cuatro usuarios estuvieron de acuerdo (4). Seis usuarios manifestaron estar totalmente de acuerdo con el fomento de la colaboración e interacción entre docentes y alumnos a través de la práctica frecuente de la guía (5) y cuatro usuarios estuvieron de acuerdo (4), de igual forma cinco usuarios estuvieron totalmente de acuerdo con la guía como modelo para la integración de entornos colaborativos al sistema educativo (5) y otros cinco usuarios estuvieron sencillamente de acuerdo con esta afirmación (4).

\section{DISCUSIÓN}

El uso que se le ha dado a los mundos virtuales refleja una significativa evolución en la educación y una tendencia por incorporarlos a las instituciones de educación superior gracias a que el potencial inherente en el uso pedagógico de estos ambientes aún no ha sido explorado completamente (Dreher et al., 2009). Una de las razones por las cuales no se han aprovechado al máximo las bondades que brindan los mundos virtuales se atribuye a los inconvenientes que se presentan en el proceso de instalación, puesta en marcha y manejo de estos entornos; es por ello que aunque se espera que tengan un gran impacto en el futuro cercano, nuestra comprensión de su uso es aún limitada (Jarmon et al., 2009).

En el estudio llevado a cabo en el año 2009 en Australia y Nueva Zelanda sobre la situación de los mundos virtuales en la educación superior se identificaron las barreras más significativas a la hora de implementar estas herramientas en el entorno universitario, entre estas sobresalen problemas de índole tecnológico traducidos en los requisitos de hardware y comunicación para un correcto funcionamiento y problemas de usabilidad representados en la curva de aprendizaje; no obstante y según el mismo estudio se pretende que estos obstáculos sean resueltos progresivamente gracias a recursos útiles como guías y manuales (Dalgarno et al., 2011), tal es el caso de la Guía Práctica Para el Uso de Second Life en la Educación Superior (Savin-Baden, 2010), que es un texto pedagógico que orienta a los docentes a utilizar este mundo virtual y que brinda una visión general de las actividades que se pueden desarrollar en la enseñanza y en la investigación.

Acorde con lo anterior y tomando como referencia la percepción de los usuarios en cuanto a que los entornos virtuales favorecen la interacción con los alumnos y que al considerar a los mundos virtuales como herramientas colaborativas sus expectativas frente a la profundización y manejo de estos son altas; se diseña una guía didáctica basada en la integración al entorno educativo de la Universidad de Cundinamarca Sede Fusagasugá del mundo virtual elegido (TED). La guía es una recopilación de ideas y actividades que contribuyen a que los docentes tengan una experiencia satisfactoria de uso y manejo del mundo virtual The Education District y que se convierte en un medio para fomentar la colaboración y al mismo tiempo animar a la exploración y utilización de los recursos que ofrece la plataforma para que la enseñanza sea participativa.

La aplicación de la guía didáctica por parte de los usuarios ejecutando diversas tareas coincide con otros casos como el de la Universidad de Texas en 2007 (Jarmon et al., 2009) y el estudio de Peterson en 2006 (Hew y Cheung, 2010); en el primero se desarrolló un ambiente de aprendizaje colaborativo con realidad virtual en el que se examinó su diseño y usabilidad; estudiantes y educadores realizaron actividades tales como subir una diapositiva de PowerPoint y usar las herramientas de comunicación; en el segundo se exploró la interacción entre estudiantes japoneses en ActiveWorlds, pidiéndoles que utilizaran el chat y que participaran en tareas como armar rompecabezas, tomar decisiones e intercambiar opiniones, al final todos los participantes respondieron favorablemente y concordaron en que los ambientes de aprendizaje virtual tienen potencial para el desarrollo de la educación.

Al medir la facilidad de uso y a pesar de que los usuarios presentaron dificultades al ejecutar ciertas tareas, la guía didáctica explica paso a paso y coherentemente las acciones que se pueden efectuar dentro del mundo virtual, esto se confirma en el nivel de utilidad en donde los usuarios expresan que la guía contiene los elementos necesarios que orientan al lector acerca de la instalación, exploración, administración y gestión de contenidos. No obstante lo anterior, es necesaria la presencia de la comunicación, colaboración y cooperación entre la comunidad académica, ya que estos factores desempeñan un papel fundamental en la posibilidad de influir en la intención de uso y aceptación (Fetscherin y Lattemann, 2008), en este sentido se 
indican algunas sugerencias que se pueden considerar con el objetivo de consolidar la aceptación y uso frecuente de la guía: 1) Destinar sesiones para despejar dudas y practicar aquellos aspectos en los cuales los usuarios presentaron dificultades; 2) Socializar y explicar la guía a los Docentes Medio Tiempo Ocasional y Hora Cátedra; 3) Sensibilizar a los alumnos sobre la importancia de participar en entornos colaborativos; 4) Compartir la guía didáctica en todos los programas de la Universidad de Cundinamarca y en otras instituciones de educación superior. A su vez y partiendo del trabajo futuro se contempla una serie de perspectivas:1) La Universidad de Cundinamarca Sede Fusagasugá se basara en un sistema de aprendizaje colaborativo; 2) Los alumnos del Programa de Administración de Empresas de la Universidad de Cundinamarca Sede Fusagasugá tendrán una visión crítica y emprendedora gracias a la simulación de situaciones generadas dentro del mundo virtual; 3) Los alumnos de la Universidad de Cundinamarca construirán conocimiento debido a la inmersión y participación en este entorno virtual de aprendizaje; 4) Con la exteriorización de la guía a otras instituciones, esta se convertirá en prototipo para la integración de otras herramientas colaborativas.

\section{CONCLUSIONES}

Teniendo en cuenta los resultados mostrados, se obtienen las siguientes conclusiones acerca de la guía didáctica: 1) Cumple con las expectativas de los usuarios; 2) Suministra las orientaciones necesarias para el manejo del mundo virtual The Education District; 3) Promueve el aprendizaje colaborativo y estimula la creatividad; 4) Es un modelo de integración de entornos colaborativos al sistema educativo de nivel superior.

\section{REFERENCIAS}

Baker, S. C., Wentz, R. K., y Woods, M. M. Using Virtual Worlds in Education: Second Life as an Educational Tool, doi: http://doi.org/10.1080/00986280802529079, Teaching of Psychology, 36(1), 59-64 (2009)

Calzadilla, M. E. Aprendizaje Colaborativo y Tecnologías de la Información y la Comunicación, http://rieoei.org/tec_edu7.htm, ISSN 1681-5653, Revista Iberoamericana de Educación, 1(10), 1-10 (2002)

Campbell, C. Learning in a different life: Pre-service education students using an online virtual world, https://journals.tdl.org/jvwr/index.php/jvwr/article/viewArticle/379, ISSN: 1941-8477, Journal for Virtual Worlds Research, 2(1) (2009)

Coffman, T., Klinger, M. 'Utilizing Virtual Worlds in Education: The Implications for Practice'. http://waset.org/publications/3382, ISSN: 1307-6892, World Academy of Science, Engineering and Technology, International Science Index 11, International Journal of Social, Behavioral, Educational, Economic, Business and Industrial Engineering, 1(11), 612-616 (2007)

Chibás-Ortíz, F., Borroto-Carmona, G., y Almeida-Santos, F. De. Gestión de la creatividad en entornos virtuales de aprendizaje colaborativos: Un proyecto corporativo de EAD, doi: http://dx.doi.org/10.3916/C432014-14, Comunicar, 22(43), 143-151 (2014)

Dalgarno, B., y Lee, M. J. W. What are the learning affordances of 3-D virtual environments? doi: http://doi.org/10.1111/j.1467-8535.2009.01038.x, British Journal of Educational Technology, 41(1), 10-32 (2010)

Dalgarno, B., Lee, M. J. W., Carlson, L., Gregory, S., y Tynan, B. Institutional support for and barriers to the use of 3D immersive virtual worlds in higher education, In Proceedings ASCILITE 2011: 28th Annual Conference of the Australasian Society for Computers in Learning in Tertiary Education: Changing Demands, Changing Directions, 316-330, Hobart, Tasmania, Australia, December 4-7 (2011)

Dalgarno, B., Lee, M. J., Carlson, L., Gregory, S., y Tynan, B, An Australian and New Zealand scoping study on the use of 3D immersive virtual worlds in higher education, doi: http://dx.doi.org/10.14742/ajet.978, Australasian Journal of Educational Technology, 27(1), 1-15 (2011)

Dreher, C., Reiners, T., Dreher, N., y Dreher, H. Virtual worlds as a context suited for information systems education: Discussion of pedagogical experience and curriculum design with reference to Second Life, http://search.proquest.com/openview/d599f94ca688bcdffa895510cea443ed/1 ?pq-origsite=gscholar, ISSN: 10553096 Journal of Information Systems Education, 20(2), 211 (2009) 
Fetscherin, M., y Lattemann, C. User acceptance of virtual worlds, http://search.proquest.com/openview/74674bc256da716109885891b67b2de4/1 ?pq-origsite=gscholar, ISSN: 1567-4223, Journal of electronic commerce research, 9(3), 231-242 (2008)

Hew, K. F., y Cheung, W. S. Use of three dimensional (3-D) immersive virtual worlds in K-12 and higher education settings: $A$ review of the research, doi: http://doi.org/10.1111/j.1467-8535.2008.00900.x, British Journal of Educational Technology, 41(1), 33-55 (2010)

Huang, H. M., Rauch, U., y Liaw, S. S., Investigating learners' attitudes toward virtual reality learning environments: Based on a constructivist approach, doi: 10.1016/j.compedu.2010.05.014 . Source: DBLP, Computers \& Education, 55(3), 1171-1182 (2010)

Jarmon, L., Traphagan, T., Mayrath, M., y Trivedi, A. Virtual world teaching, experiential learning, and assessment: An interdisciplinary communication course in Second Life, doi: http://dx.doi.org/10.1016/j.compedu.2009.01.010, Computers \& Education, 53(1), 169-182 (2009)

Lizarralde, F. A. y Huapaya, C. R. Análisis de una Plataforma Virtual 3-D Descentralizada para el Desarrollo de Simulaciones Educativas, doi: http://dx.doi.org/10.4067/S0718-50062012000600002, Formación Universitaria, 5(6), 3-12 (2012)

Moldoveanu, A., Gradinaru, A., Ferche, O. M., y Stefan. L, the 3D UPB mixed reality campus: Challenges of mixing the real and the virtual. Proceedings of the 18th International Conference on System Theory, Control and Computing, 538-543, Sinaia, Romania, October 17-19 (2014)

Quinche, J. C., y González, F. L. Entornos Virtuales 3D, Alternativa Pedagógica para el Fomento del Aprendizaje Colaborativo y Gestión del Conocimiento en Uniminuto, doi: http://dx.doi.org/10.4067/S071850062011000200006, Formación Universitaria, 4(2), 45-54 (2011)

Salinas, J; Benito, B d. Los entornos tecnológicos en la universidad, http://www.redalyc.org/articulo.oa?id=36803206, ISSN: 1133-8482, Pixel-Bit. Revista de Medios y Educación, (32), 83-100 (2008)

Salinas, J y Lizana, A. Competencias docentes para los nuevos escenarios de aprendizaje, https://dialnet.unirioja.es/servlet/articulo?codigo=4840056, ISSN: 0213-8646, Revista interuniversitaria de formación del profesorado, (79), 145-163 (2014)

Savin-Baden, M, A practical guide to using Second Life in higher education, 1aㅡ edición, 1-207, McGraw-Hill Education (UK) (2010)

Whitton, N., y Hollins, P. Collaborative virtual gaming worlds in higher education, doi: http://doi.org/10.1080/09687760802526756, Research in learning technology, 16(3), 221-229 (2008) 